\title{
Toll-like receptor 2 deficiency improves insulin sensitivity and hepatic insulin signalling in the mouse
}

\author{
L.-H. Kuo • P.-J. Tsai • M.-J. Jiang • Y.-L. Chuang • \\ L. Yu • K.-T. A. Lai $\cdot$ Y.-S. Tsai
}

Received: 5 August 2010 /Accepted: 1 September 2010 /Published online: 22 October 2010

(C) Springer-Verlag 2010

\begin{abstract}
Aims/hypothesis Substantial evidence suggests a link between elevated inflammation and development of insulin resistance. Toll-like receptor 2 (TLR2) recognises a large number of lipid-containing molecules and transduces inflammatory signalling in a variety of cell types, including insulin-responsive cells. Considering the contribution of the fatty acid composition in TLR2-depedent signalling, we hypothesised that the inflammatory signals transduced by TLR2 contribute to insulin resistance.
\end{abstract}

Electronic supplementary material The online version of this article (doi:10.1007/s00125-010-1931-5) contains supplementary material, which is available to authorised users.

L.-H. Kuo $\cdot$ Y.-S. Tsai $(\bowtie)$

Institute of Basic Medical Sciences, College of Medicine,

National Cheng Kung University,

Tainan, Taiwan, Republic of China

e-mail: yaustsai@mail.ncku.edu.tw

P.-J. Tsai

Department of Medical Laboratory Science and Biotechnology,

College of Medicine, National Cheng Kung University,

Tainan, Taiwan, Republic of China

M.-J. Jiang $•$ Y.-L. Chuang $•$ Y.-S. Tsai

Institute of Clinical Medicine and Cardiovascular Research

Center, College of Medicine and Hospital,

National Cheng Kung University,

Tainan, Taiwan, Republic of China

L. Yu

Institute of Behavioral Medicine, College of Medicine,

National Cheng Kung University,

Tainan, Taiwan, Republic of China

K.-T. A. Lai

NovoTaiwan Biotech,

Taipei, Taiwan, Republic of China
Methods Mice deficient in TLR2 were used to investigate the in vivo roles of TLR2 in initiating and maintaining inflammation-associated insulin resistance and energy homeostasis.

Results We first recapitulated the observation with elevated expression of TLR2 and inflammatory cytokines in white adipose tissue and liver of $o b / o b$ mice. Aged or high-fat-fed TLR2-deficient mice were protected from obesity and adipocyte hypertrophy compared with wild-type mice. Moreover, mice lacking TLR2 exhibited improved glucose tolerance and insulin sensitivity regardless of feeding them regular chow or a high-fat diet. This is accompanied by reductions in expression of inflammatory cytokines and activation of extracellular signal-regulated kinase (ERK) in a liver-specific manner. The attenuated hepatic inflammatory cytokine expression and related signalling are correlated with increased insulin action specifically in the liver in TLR2-deficient mice, reflected by increased insulinstimulated protein kinase B (Akt) phosphorylation and IRS1 tyrosine phosphorylation and increased insulinsuppressed hepatocyte glucose production.

Conclusions/interpretation The absence of TLR2 attenuates local inflammatory cytokine expression and related signalling and increases insulin action specifically in the liver. Thus, our work has identified TLR2 as a key mediator of hepatic inflammation-related signalling and insulin resistance.

Keywords Extracellular signal-regulated kinase . Inflammation Insulin resistance $\cdot$ Toll-like receptor 2

\author{
Abbreviations \\ Akt Protein kinase B \\ BAT Brown adipose tissue \\ ERK Extracellular signal-regulated kinase
}




$\begin{array}{ll}\text { HF } & \text { High fat } \\ \text { IKK } & \text { IkB kinase } \\ \text { ITT } & \text { Insulin tolerance test } \\ \text { JNK } & \text { c-Jun N-terminal kinase } \\ \text { MCP-1 } & \text { Monocyte chemotactic protein-1 } \\ \text { NF-kB } & \text { Nuclear factor kappa B } \\ \text { PI3K } & \text { Phosphoinositide 3-kinase } \\ \text { RC } & \text { Regular chow } \\ \text { TLR } & \text { Toll-like receptor } \\ \text { WAT } & \text { White adipose tissue }\end{array}$

\section{Introduction}

Insulin resistance is the chief abnormality present in metabolic syndrome. Increasing evidence suggests causative links between inflammation and development of insulin resistance $[1,2]$. Thus, the inflammatory nuclear factor kappa B (NF-KB) signalling pathway is activated and pro-inflammatory cytokines are produced in the white adipose tissue (WAT) and livers of insulin-resistant animals and humans [3-5]. Genetic or pharmacological inhibition of NF-KB pathways and cytokine neutralisation reverses insulin resistance $[4,6]$. Previous studies demonstrated that inflammation-induced activation of protein kinases, such as IKB kinases (IKKs), c-jun N-terminal kinases (JNKs) and extracellular signal-regulated kinases (ERKs), inhibits tyrosine phosphorylation of IRS1 and suppresses downstream insulin signalling. This results in inefficient glucose uptake and use [7-9]. Thus, it is critical to identify the target(s) and mechanism(s) that initiate inflammatory responses leading to insulin resistance.

Insulin resistance is frequently associated with obesity [10] and dyslipidaemia [11]. Obesity is thought to be the most important identified factor contributing to insulin resistance [12]. Dyslipidaemia, characterised by abnormal amounts and composition of lipids and/or lipoproteins in blood, represents an additional risk factor for insulin resistance. NEFAs and triacylglycerols are elevated in obese and diabetic individuals and animals. Previous studies demonstrated that exposure to elevated NEFA causes activation of inflammatory signals and production of cytokines in multiple cell types, and these findings have been correlated with impairments of insulin actions [13, 14]. Thus, circulating lipid and associated inflammation appear to be a link between nutrient excess and insulin resistance. Recent studies have suggested that the induction of inflammatory pathways by dietary factors may mediate through the membrane receptors, including toll-like receptors (TLRs) [15].

TLRs play a central role in the innate immune response by recognising conserved patterns in diverse pathogenic molecules and activating pro-inflammatory signalling pathways $[16,17]$. TLR2, a subclass of TLRs, is the main receptor recognising components of bacterial cell wall, lipoproteins and lipopeptides. Ligand-induced dimerisation of TLR2 with either TLR1 or TLR6 triggers recruitment of adaptor proteins, following a cascade of kinase activation, ultimately leading to activation of NF$\mathrm{KB}$ and production of pro-inflammatory cytokines. The binding specificity of TLR2 with lipoprotein ligand is mediated through its dimerisation with either TLR1 or TLR6. The TLR2-TLR1 complex recognises triacylated lipoprotein, whereas the TLR2-TLR6 complex senses diacylated lipoprotein [18]. Thus, the number of acyl chain of lipoproteins is finely differentiated by TLR1TLR2 and TLR2-TLR6. In addition to the number of acyl chains, other characteristics, such as length and ester bond of acyl chains, are critical for the biological activity of TLR2-dependent signalling [19, 20]. These results suggest that the fatty acid composition plays a central role in ligand recognition and receptor activation for TLR2. Furthermore, other studies demonstrated that TLR2 is involved in NEFA-induced insulin resistance [21-23]. It is reasonable to postulate that the fatty acid moiety from nutrients could potentially activate TLR2 and transduce the inflammatory signals.

Although the predominant site of TLR2 production is on cells of the innate immune system [17], TLR2 is found on a number of insulin-responsive cells, including adipose, skeletal muscle and liver cells [24, 25]. However, the roles of TLR2 in initiating and maintaining inflammatoryassociated insulin resistance and energy homeostasis in vivo have not been established. Here, we show that mice with TLR2 deficiency are protected from developing insulin resistance and adiposity. The increased insulin sensitivity is associated with increased insulin-signal transduction, improved glucose metabolism, and reduced inflammatory cytokine expression and related signalling specifically in the liver.

\section{Methods}

Mice Mice deficient in TLR2 $\left(\mathrm{Tlr}^{-/-}\right)$were kindly provided by S. Akira [26] and maintained on a C57BL/6 genetic background. Studies were carried out using both male and female $T l r 2^{-/}$mice and age-matched wild-type (WT) C57BL/6 mice, obtained from National Cheng Kung University Laboratory Animal Center. Leptin-deficient $(\mathrm{ob} /$ $o b)$ mice were obtained from the Jackson Laboratory (Bar Harbor, ME, USA). Mice were fed ad libitum either regular chow (RC) (Purina Laboratory Rodent Diet 5001, PMI Nutrition International, Richmond, IN, USA) or a high-fat (HF) diet (58Y1; TestDiet, Richmond, IN, USA). 
Tissue collection and RNA analysis Tissues were collected and stored in RNAlater (Ambion, Austin, TX, USA), and RNA was extracted using the TRIzol Reagent (Invitrogen, Carlsbad, CA, USA). Samples of mRNA were analysed with SYBR Green-based real-time quantitative RT-PCR (Applied Biosystems, Foster City, CA, USA), with $\beta$-actin or cyclophilin $\mathrm{A}$ as the reference gene in each reaction. Sequences of the primers used for RT-PCR assays are shown in Electronic supplementary material (ESM) Table 1.

Protein analysis For the insulin signalling, $62.5 \mathrm{mU} / \mathrm{kg}$ insulin was administered through the portal vein, and muscle tissues were collected $5 \mathrm{~min}$ after injection as described by Hirosumi et al. [27]. Liver and WAT tissue samples were collected 2 min after injection with 200 and $500 \mathrm{mU} / \mathrm{kg}$ insulin through the portal vein, respectively. Further methods can be found in the ESM.

\section{Results}

Upregulation of TLR2 and inflammatory cytokines in WAT and liver of obese mice To examine the association of inflammation with obesity and insulin resistance, we determined the gene expression of inflammatory mediators in the major insulin-responsive tissues of $o b / o b$ mice. We found that mRNA expression of Tnf was significantly elevated in WAT and liver, but not in muscle, of $o b / o b$ mice, compared with those of WT controls (Fig. 1a). Expression of $I l 1 b$ and $I l 6$ mRNA was increased in WAT, but not in liver and muscle, of $o b / o b$ mice. The upregulation of inflammatory genes was accompanied by significantly increased expression of TLR2 and moderate induction of TLR4 expression as shown in levels of both mRNA and protein (Fig. 1b,c). Consistent with this, the upregulation of TLR2 was evident in WAT and liver, but not in muscle. These results imply that obesity and nutrient excess elicit upregulation in expression of inflammatory cytokines and TLR2 predominantly in WAT and liver. This prompted us to test directly whether TLR2 and its induced inflammation are involved in metabolic disturbances.

Increased insulin sensitivity in Tlr $2^{-/}$mice To investigate the role of TLR2 in glucose homeostasis, we examined the plasma levels of glucose and insulin in $T l r 2^{-/-}$and WT mice. Plasma glucose and insulin levels after fasting were both significantly lower in $T l r 2^{-/}$mice than in those of RC-fed WT mice (Fig. 2a,b). Consistent with these findings, $T l r 2^{-/}$mice fed an HF diet had significantly lower glucose and insulin levels relative to their WT controls. To assess the effect of TLR2 deficiency on the whole-body glucose utilisation, we performed an OGTT. $T l r 2^{-/}$mice cleared glucose faster than WT mice, regardless of diet, indicating improved glucose tolerance in $T l r 2^{-/}$mice (Fig. 2c,d). This improved glucose tolerance was accompanied by significantly decreased plasma insulin levels during OGTT of $\mathrm{Tlr}^{-/}$mice, regardless of diet (Fig. 2e,f). Thus, the insulin-resistance index calculated from the OGTT was significantly lower in $T l r 2^{-/}$mice relative to their WT controls with either diet (Fig. 2g). Consistent with this, insulin tolerance tests (ITT) showed that glucose-lowering effects of insulin were improved in $T l r 2^{-/}$mice regardless of diet (Fig. 2h,i). These results demonstrated that the absence of TLR2 increases glucose tolerance and insulin sensitivity regardless of feeding mice $\mathrm{RC}$ or an $\mathrm{HF}$ diet.

Decreased body weight and fat mass in Tlr $2^{-/}$mice We next asked whether loss of TLR2 alters energy homeostasis in mice. The growth curve of $T l r 2^{-1-}$ mice was the same as that of WT mice when mice were fed RC, but significantly differed in mice fed the HF diet (Fig. 3a,b). Body composition analysis by echoMRI identified significantly
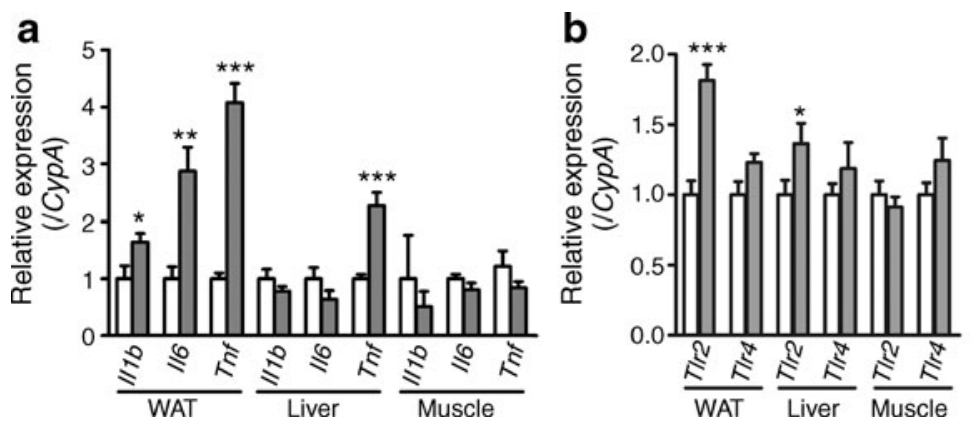

C

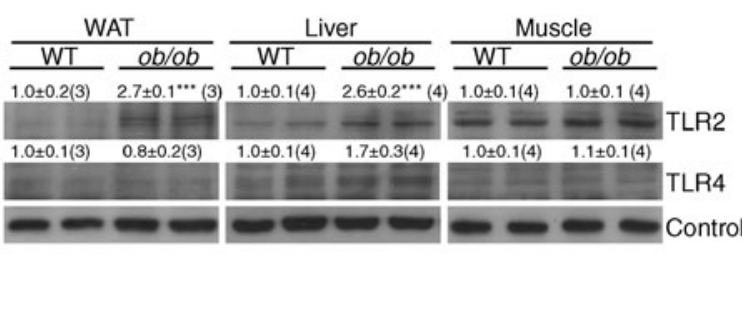

Fig. 1 Expression of inflammatory cytokines and TLRs in WAT, liver and gastrocnemius muscle of $o b / o b$ mice. (a) $I l l b$, Il6 and Tnf, and (b) Tlr2 and Tlr4 mRNA levels in tissues from male $o b / o b$ mice $(n=6-8)$ relative to samples from WT mice $(n=6-10)$. c Protein levels of TLR2 and TLR4 in tissues from male $o b / o b$ and WT mice. Samples from representative animals are shown in the western blot, with each lane representing one animal. The intensities of the bands, quantified densitometrically relative to WT, are shown with sample number in parentheses. White bars, WT; grey bars, ob/ob. ${ }^{*} p<0.05,{ }^{* *} p<0.01$ and $* * * p<0.001$ compared with WT mice 
Fig. 2 Glucose homeostasis and insulin sensitivity in $\mathrm{Tlr}^{-/-}$ mice. a Fasting plasma glucose in male mice fed $\mathrm{RC}$ and $\mathrm{HF}$ diets (WT RC, $n=15$; Tlr $2^{-/}$ $\mathrm{RC}, n=16$; WT HF, $n=16$; $T l r 2^{-/}$HF, $n=12$ ). b Fasting insulin levels in male mice fed $\mathrm{RC}$ and HF diets (WT RC, $n=8$; $T l r 2^{-/}$RC, $n=7$; WT HF, $n=$

14; Tlr2 $\left.2^{-/} \mathrm{HF}, n=9\right)$. (c,d) Plasma glucose during OGTT in male mice fed (c) RC and (d) HF diets. (e,f) Insulin levels during OGTT in male mice fed (e) RC and (f) HF diets. $\mathbf{g}$ Insulin-resistance (IR) index during OGTT in male mice fed RC and HF diets. (h,i) ITT results for male mice fed (h) RC and (i) HF diets. White bars/symbols, WT; black bars/symbols, $T l r 2^{--}{ }^{*}{ }^{*} p<$ $0.05,{ }^{* *} p<0.01$ and $* * * p<0.001$ compared with WT mice
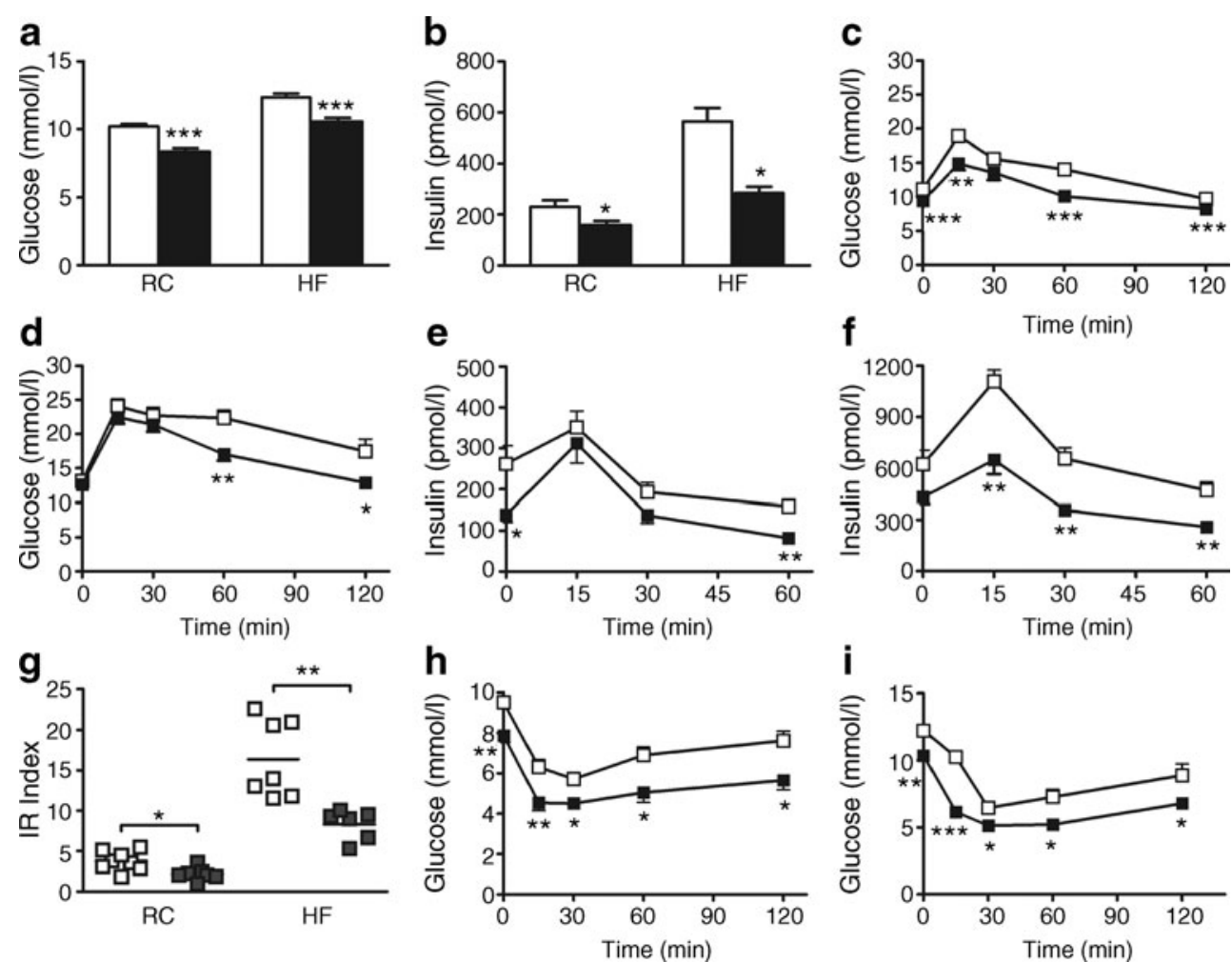

reduced fat mass and increased lean mass in aged (11 month old) $T l r 2^{-1}$ mice, while no difference in fat and lean mass was found between young ( 2 month old) $T l r 2^{-/}$and WT mice (Fig. 3c). Furthermore, we found significant decreases in individual fat mass of aged Tlr $2^{-/-}$mice (Fig. 3d). The intra-abdominal, including gonadal and retroperitoneal, WAT masses in $T l r 2^{-/}$mice were about $35 \%$ of those in WT mice. A similar reduction was also observed in the subcutaneous inguinal WAT mass of $T l r 2^{-/-}$mice, although the difference did not reach statistical significance. The interscapular brown adipose tissue (BAT) weight of $T l r 2^{-1-}$ mice was about $70 \%$ that of WT mice. The weights of major organ, including kidney, liver, spleen and heart, were indistinguishable between genotypes. Gastrocnemius skeletal muscle weight of $\mathrm{Tlr}^{-2^{--}}$mice was significantly increased. These data indicate that the absence of TLR2 causes reductions in fat deposition and content. While HF feeding increased body weight of WT mice, the increases in body weight of $T l r 2^{--}$mice were significantly diminished (Fig. 3b). Consistent with this, the individual WAT and BAT masses were significantly less in $T l r 2^{-/}$mice fed an HF diet than in WT mice (Fig. 3e).

Microscopically, adipocytes in gonadal WAT of RC-fed aged $T l r 2^{-/}$mice were smaller than WT cells (mean area $821 \mu^{2}$ in $T l r 2^{-/-}$vs $1503 \mu \mathrm{m}^{2}$ in WT) (Fig. 3f,g). The numbers of adipocytes were similar in the Tlr $2^{--}$and WT mice $\left(1.89 \times 10^{7}\right.$ in $\mathrm{TLR}^{-/-}$vs $1.73 \times 10^{7}$ in WT). HF feeding led to fat deposition in both $T l r 2^{-/}$and WT mice and the difference in adipocyte size between genotypes was

preserved (mean area $2242 \mu \mathrm{m}^{2}$ in $T l r 2^{-/-}$vs $3276 \mu \mathrm{m}^{2}$ in WT) (Fig. 3h,i). The decrease in adipocyte size of $T l r 2^{-/}$ mice under HF feeding was associated with a decrease in the number of adipocytes $\left(1.21 \times 10^{7}\right.$ in $T l 2^{-/}$vs $2.62 \times$ $10^{7}$ in WT). Thus, the deficiency of TLR2 brought a shift in the distribution of adipocyte sizes towards smaller adipocytes in WAT (Fig. 3g,i). The reduction in fat mass of $\mathrm{Tlr}^{-/-}$mice appears to result primarily from the reduction in adipocyte size with a modest decrease in adipocyte number. In vitro differentiation of embryonic fibroblasts showed that adipogenic ability judged by the formation of cells staining positive with Oil red $\mathrm{O}$ after hormone stimulation was preserved in $T l r 2^{-/}$mice (Fig. 3j), ruling out the possibility of defective adipogenic programming with TLR2 deficiency.

The critical variables contributing to body weight maintenance include energy intake and expenditure. Metabolic analysis showed that $\mathrm{Tlr}^{-/-}$mice had similar daily food intake to WT mice (Fig. 4a), suggesting that $T l r 2^{-/-}$ mice have normal energy absorption. Body temperature was modestly increased in male and significantly increased in female $\mathrm{Tlr}^{-/-}$mice, regardless of diet (Fig. 4b). Basal energy expenditure, as measured by oxygen consumption $\left(\dot{V} \mathrm{O}_{2}\right)$, during the dark phase was dramatically increased in $T l r 2^{--}$mice (Fig. 4c,d). As the increases in $\dot{V} \mathrm{O}_{2}$ and $\dot{V} \mathrm{CO}_{2}$ were coordinated, the respiratory exchange ratio was unchanged (Fig. 4e,f). Consistent with this, vertical rearing of both young and aged $T l r 2^{-/}$mice during the dark phase was markedly increased (Fig. 4g). Ambulatory locomotor 

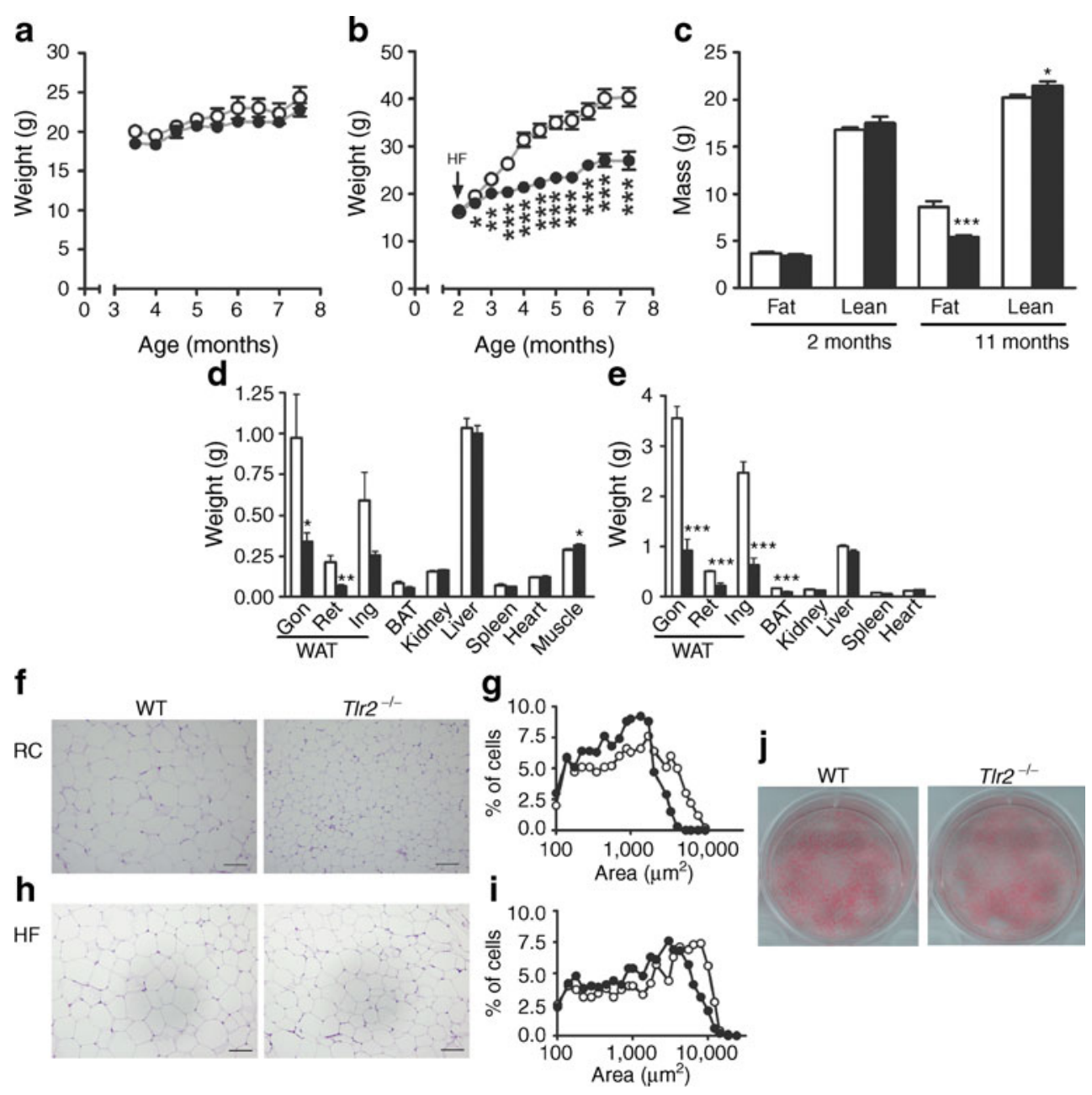

Fig. 3 Body composition of $T l r 2^{-/-}$mice. (a,b) Body weights of female mice fed (a) RC (WT, $n=8 ; T l r 2^{-/}, n=7$ ) and (b) HF diets (WT, $n=7 ; T l r 2^{-/-}, n=7$ ); at first time point after HF was started, $p<$ 0.05 ; at time point $2, p<0.01$; at all subsequent time points, $p<0.001$. c Body composition analyses of 2 and 11 month old male mice by MRI (2 month old mice, $n=5$; WT 11 month old mice, $n=7$; $T l r 2^{-/}$ 11 month old mice, $n=9)$. (d,e) Organ weights in (d) 11 month old female WT $(n=8)$ and $T l r 2^{--}(n=7)$ mice fed RC and (e) 7 month old female WT $(n=7)$ and $T l r 2^{-/-}(n=7)$ mice fed the HF diet. Gon,

activity of aged $T l r 2^{-/-}$mice during both the dark and light phases was significantly increased (Fig. 4h). Thus, energy dissipation appeared increased in $T l r 2^{-/}$mice.

Lipid profile and inflammatory mediators in Tlr2 $2^{-/-}$ mice WAT actively secretes signalling molecules, including NEFA, adipokines and inflammatory molecules, into the circulation and communicates with other organs to regulate insulin sensitivity [28]. Fasting plasma triacylglycerol and NEFA levels were not distinguishable between genotypes in mice fed $\mathrm{RC}$, and were significantly lower in $\mathrm{Tlr} 2^{-/-}$ mice than in WT mice fed an HF diet (Fig. 5a,b). Plasma cholesterol levels were not different between genotypes, regardless of diet (Fig. 5c). Circulating leptin and resistin gonadal WAT; Ing, inguinal WAT; Ret, retroperitoneal WAT. Muscle indicates gastrocnemius skeletal muscle. (f,h) Morphology and (g,i) cell size distribution of gonadal WAT from WT and $T l r 2^{--}$mice fed (f,g) RC and (h,i) HF diets. The scale bar indicates $100 \mu \mathrm{m}$ for all images. $\mathbf{j}$ In vitro adipogenesis in embryonic fibroblasts. Oil red $\mathrm{O}$ staining of plates after 8 days of differentiation. White bars/symbols, WT; black bars/symbols, Tlr $2^{-/-}$. ${ }^{*} p<0.05,{ }^{* *} p<0.01$ and $* * * p<$ 0.001 compared with WT mice

levels were not distinguishable between genotypes in mice fed RC, but were reduced in $T l r 2^{-/-}$mice fed an HF diet (Fig. 5d,e). Adiponectin levels in circulation were comparable between $T l r 2^{-/}$and WT mice, regardless of diet (Fig. 5f). While circulating inflammatory molecules, including IL-6, monocyte chemotactic protein-1 (MCP-1) and TNF- $\alpha$, were higher in mice fed an HF diet than in those fed $\mathrm{RC}$, no difference was detectable between genotypes, regardless of diet (Fig. 5g-i). Although the decreased levels of plasma triacylglycerol, NEFA and resistin may account for increased insulin sensitivity in $T l r 2^{-/-}$mice upon HF feeding, the increased insulin sensitivity was, however, observed in $T l r 2^{-/}$mice fed RC despite any significant change in plasma triacylglycerol, NEFA and resistin. These 

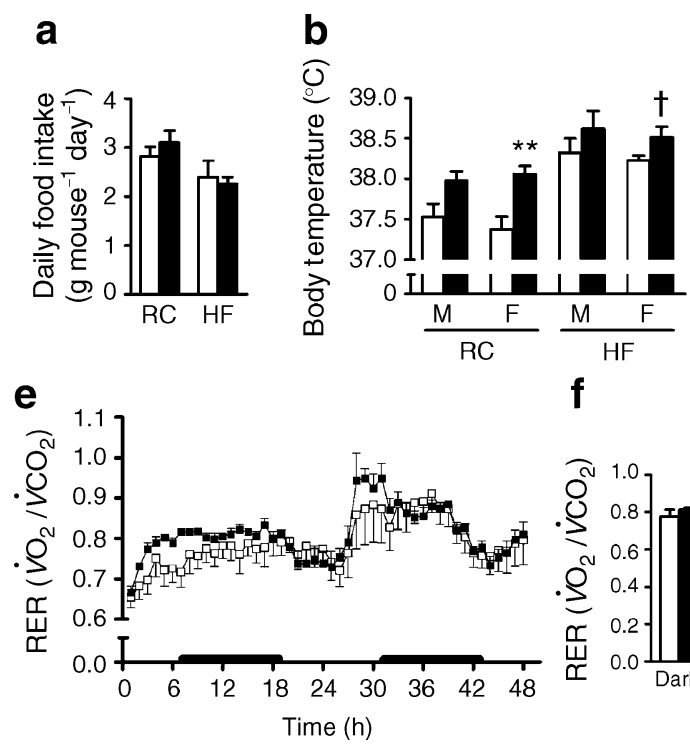

f

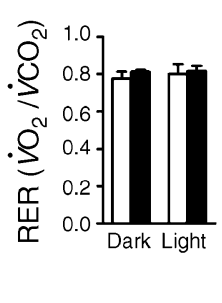

Fig. 4 Energy homeostasis in Tlr $2^{-/}$mice. a Daily food intake in metabolism cages ( $n=5$ for all results). b Body temperature measured at 10:00 hours in WT and Tlr $2^{-/-}$mice (RC male [M] mice, $n=8$; WT RC female $[\mathrm{F}]$ mice, $n=8$; $T l r 2^{-\perp}$ F mice, $n=7$; WT HF M mice, $n=8$; $T l r 2^{--}$M mice, $n=5$; WT HF F mice, $n=7$; $T l r 2^{-/}$F mice, $n=7$. (c) Oxygen consumption $\left(\dot{V} \mathrm{O}_{2}\right)$ and (d) mean AUC; (e) respiratory exchange ratio (RER) and (f) mean $12 \mathrm{~h}$ values, of 2 month old male mice in indirect calorimetry over $48 \mathrm{~h}$ (WT, $\left.n=5 ; \operatorname{Tl} r 2^{-/}, n=4\right)$. Bold

results suggest that the deficiency of TLR2 has limited effects on systemic levels of inflammatory mediators.

Attenuated local inflammatory cytokine expression and signalling in Tlr $2^{--}$mice Although the deficiency of TLR2 did not elicit apparent differences in the systemic levels of inflammatory molecules, insulin sensitivity can be affected through the change in local insulin-responsive tissues. We next examined whether the absence of TLR2 would attenuate local inflammation. Tlr $2^{-/-}$RC-fed mice exhibited, in liver, a significant reduction in expression of $I l 6$ and a tendency towards decreased $I l 1 b$ expression, but no change in expression of Tnf and macrophage marker Emrl (Fig. 6a). No difference in expression of these cytokines was identified in WAT and muscle of $T l r 2^{-/-}$mice. Interestingly, dramatic reductions in expression of $I l 1 \mathrm{l}, \mathrm{Il} 6$ and $E m r l$ as well as a tendency towards a decrease in expression of $\operatorname{Tnf}$ were observed in WAT and liver of Tlr $2^{-/}$mice fed the HF diet (Fig. 6b). Expression of these genes, except Tnf, remained unaltered in muscle of $\mathrm{Tlr}^{-/}$HF-fed mice (Fig. 6b). Because TLR2 has been demonstrated to transduce signal to activate protein kinases, including IKK, JNK and ERK [79], we examined their phosphorylation in the insulinresponsive tissues. No difference in phosphorylation of IKK $\alpha / \beta$ and JNK between WT and $T l r 2^{-/}$mice was detectable in WAT, liver and muscle, regardless of diet (Fig. 6c,d). While phosphorylation of ERK1/2 was similar in WAT and muscle in the WT and Tlr2 $2^{-/}$mice fed RC, its d

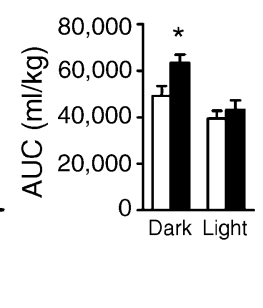

h
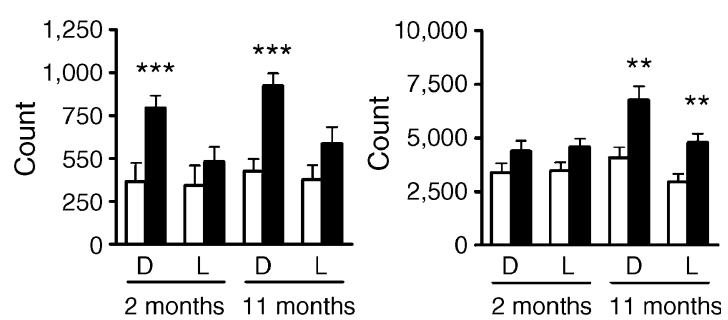

bars on the $x$-axes represent the dark phases. (g, h) Locomotor activity measured as (g) the vertical rearing and (h) the ambulatory activity, across a 30 min period of observation ( 2 month old mice, $n=5$; WT 11 month old mice, $n=7$; Tlr $2^{-/} 11$ month old mice, $n=9$; $\mathrm{D}$, dark; L, light). White bars/symbols, WT; black bars/symbols, $T l r 2^{-1-}{ }^{*} p<$ $0.05, * * p<0.01, * * * p<0.001$ and ${ }^{\dagger} p=0.0695$ compared with WT mice

phosphorylation was markedly decreased in the liver of $\mathrm{Tlr}^{-/}$mice (Fig. 6c). The decrease in hepatic ERK1/2 phosphorylation was not accompanied by the change of ERK1/2 protein content in the liver. Furthermore, the liverspecific decrease in ERK1/2 phosphorylation was also observed in Tlr $2^{-/}$mice fed the HF diet (Fig. 6d). Thus, the absence of TLR2 attenuates basal inflammatory cytokine expression and signalling specifically in the liver.

Inflammation-induced activation of IKK, JNK and ERK has been shown to phosphorylate IRS1 at its serine residue (s), which further interrupts tyrosine phosphorylation on IRS1 and suppresses insulin signalling [7-9]. To further evaluate whether the attenuated inflammatory cytokine expression and signalling in the liver of $T l r 2^{-/}$mice correlates with serine phosphorylation of IRS1, we detected the phosphorylation at Ser307 and Ser612 of IRS1. No difference in phosphorylation at Ser307 and Ser612 of IRS1 between WT and Tlr $2^{--}$livers was detected, regardless of diet (Fig. 6e,f). These results suggest that TLR2 deficiency did not affect phosphorylation at Ser307 or Ser612 of IRS1 in the liver.

Improved insulin signalling specifically in the liver of $T l r 2^{-/}$mice To determine which insulin-responsive tissue(s) exhibited improved insulin signalling in $\mathrm{Tlr}^{-/-}$mice, we examined the insulin action in mice fed RC. Insulinstimulated phosphorylation at Ser473 of protein kinase B (Akt) was increased in liver, but not in WAT and muscle of 
a
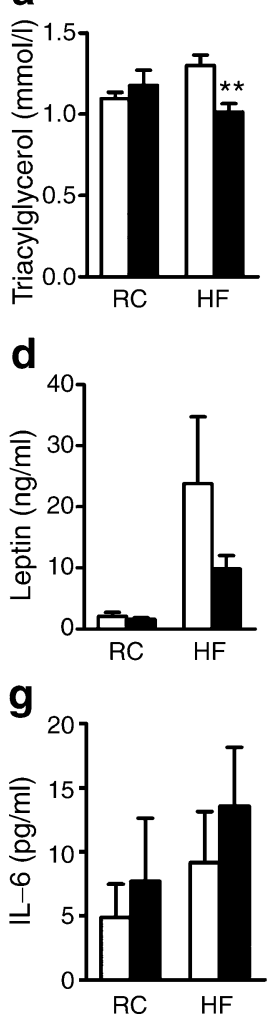

b

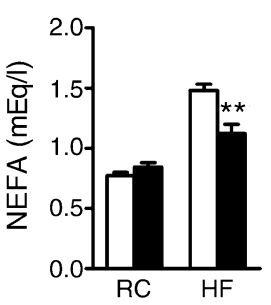

e

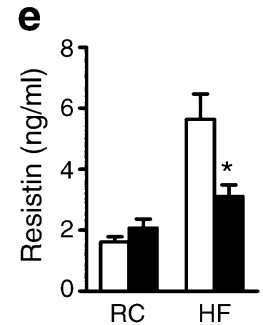

h

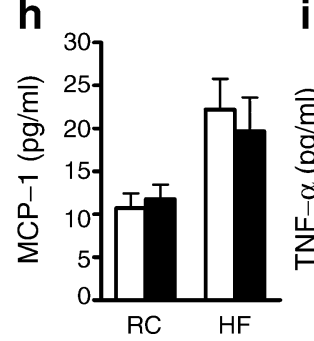

C

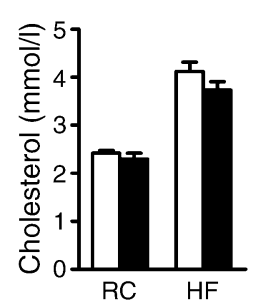

f

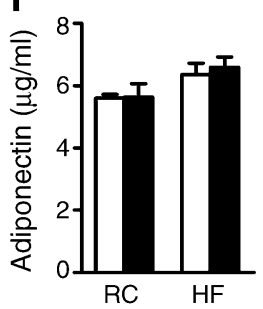

i

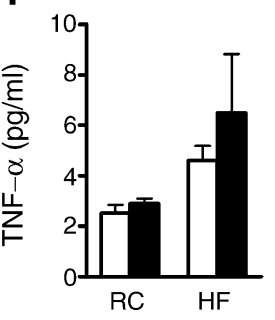

Fig. 5 Lipid profiles and inflammatory mediators in $T l r 2^{-/}$mice. Plasma levels of (a) triacylglycerol, (b) NEFA, (c) cholesterol, (d) leptin, (e) resistin, (f) adiponectin, (g) IL-6, (h) MCP-1 and (i) TNF- $\alpha$ in male mice fed RC and HF diets (WT, $n=8 ; T l r 2^{-/}, n=5$ ). White bars, WT; black bars, Tlr $2^{-/}$. ${ }^{*} p<0.05$ and ${ }^{*} p<0.01$ for $T l r 2^{-/-}$ mice compared with WT mice

$\operatorname{Tr} 2^{-/-}$mice, compared with those of WT mice (Fig. 7a). This was accompanied by increased tyrosine phosphorylation of IRS1 and association of IRS1: phosphoinositide 3kinase (PI3K) complex in the livers of $T l 2^{-/-}$mice after insulin stimulation. However, the increases in IRS1 tyrosine phosphorylation and IRS1:PI3K association were not found in WAT and muscle of $T l r 2^{-/}$mice. Consistently, the liverspecific increases in insulin-stimulated Akt phosphorylation, IRS1 tyrosine phosphorylation and IRS1:PI3K association (data not shown) were also observed in $T l r 2^{-/-}$mice fed HF diet (Fig. 7b). Thus, liver appears to be the major tissue with increased insulin signalling in $T l r 2^{-/}$mice.

Improved glucose metabolism in the liver of Tlr2 $2^{-1-}$ mice To determine the functional correlates of increased insulin signalling in the livers of $\mathrm{Tlr}^{-/-}$mice, we determined aspects of hepatic glucose metabolism. Pyruvate tolerance tests showed that glucose levels were significantly lower in $\mathrm{Tlr}^{-/}$mice in response to the administration of pyruvate, a major gluconeogenesis substrate (Fig. 8a). We next assessed the effect of TLR2 deficiency in primary hepatocytes isolated from $\mathrm{Tlr}^{-/-}$and

WT mice. TLR2 deficiency significantly reduced pyruvateinduced glucose release into the medium by primary hepatocytes (Fig. 8b). To examine the functional effects of TLR2 deficiency on hepatocyte insulin responsiveness, the ability of insulin to inhibit hepatocyte glucose production was determined. Glucose production by WT hepatocytes was reduced by $\sim 20 \%$ within this time period in response to insulin (Fig. 8c). The inhibition of glucose secretion by insulin in $T l r 2^{--}$hepatocytes was significantly increased by $50 \%$ in comparison with WT hepatocytes. Thus, increased insulin signalling secondary to TLR2 deficiency resulted in an enhancement of insulin to suppress hepatocyte glucose secretion. Together, these results indicate $T l r 2^{-\digamma}$ mice have reduced hepatic glucose production and increased response to insulin.

To determine the insulin sensitivity in the skeletal muscle, we performed the in vitro glucose uptake assay. Basal glucose uptake in muscle in vitro was similar between $T l r 2^{-/-}$and WT mice (Fig. 8d). Insulinstimulated glucose uptake in muscle of $T l r 2^{-/}$mice was 1.6 times those of WT, although the difference did not reach the statistical significance. Consistent with this, triacylglycerol content in liver, which is associated with hepatic insulin resistance, was significantly reduced in $\mathrm{Tlr}^{-/-}$mice, while muscle triacylglycerol content was not altered in $T l r 2^{-/}$mice (Fig. 8e).

\section{Discussion}

Substantial evidence indicates inflammation as a key mechanism linking metabolic disturbance to nutrient excess. To establish this link, we hypothesised that TLR2 transduces the inflammatory signals and further contributes to insulin resistance. Our findings recapitulated the observation that obesity is associated with elevated expression of inflammatory cytokines in WAT and liver. Moreover, mice lacking TLR2 exhibited enhanced insulin sensitivity, which is accompanied by increased transduction of insulin signal, improved glucose metabolism, and attenuated inflammatory cytokine expression and related signalling specifically in the liver. Thus, our work has identified TLR2 as a key mediator of hepatic inflammation-related signalling and insulin resistance.

Body weight represents the homeostasis between energy intake and expenditure. The deficiency of TLR2 caused a reduction in body weight and fat mass without alteration in food intake. Increased energy expenditure and locomotor activity, as well as body temperature, point to increased energy dissipation in $T l r 2^{-/-}$mice. It is known that increased physical activity can influence the insulin sensitivity of skeletal muscle, which is consistent with our findings of a modest increase of insulin- 


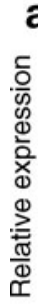
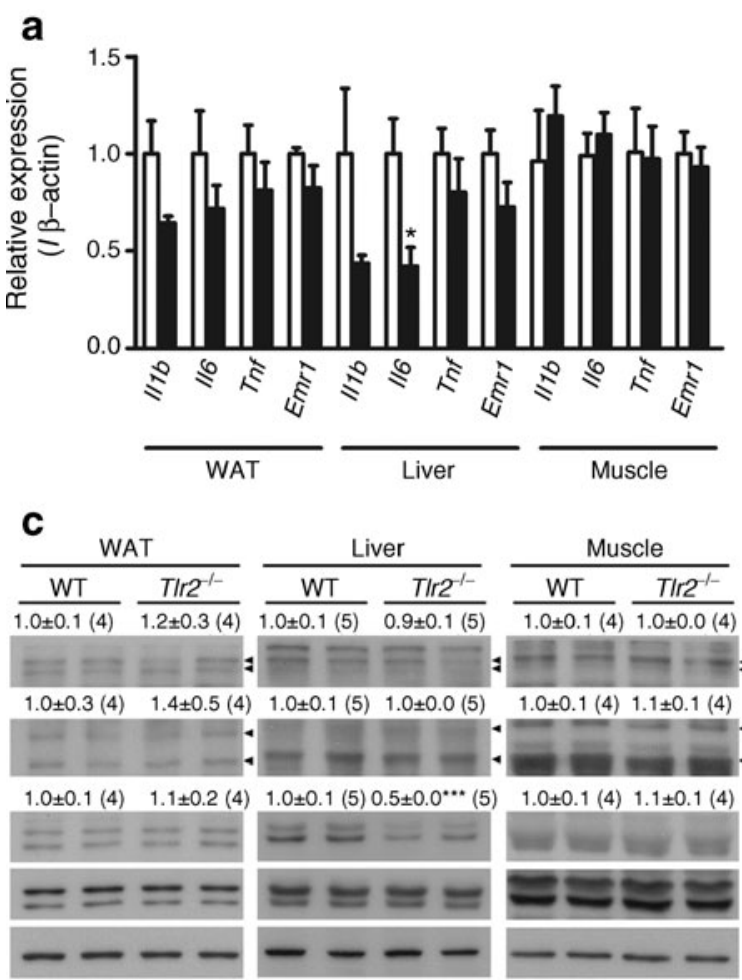

$\frac{\text { Liver }}{\text { WT } \frac{\text { TIr } 2^{1-}}{0.9 \pm 0.1(5)}}$

$\frac{\text { Muscle }}{\frac{W T}{1.0 \pm 0.1(4)} \frac{T l r 2^{-1-}}{1.0 \pm 0.0(4)}}$
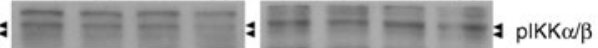

$1.0 \pm 0.1(5) \quad 1.0 \pm 0.0(5)$

$1.0 \pm 0.1(4) 1.1 \pm 0.1(4)$
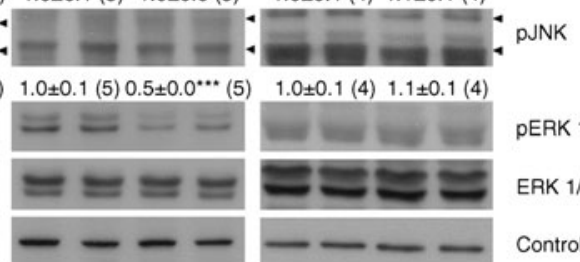

$1.0 \pm 0.1(4) 1.1 \pm 0.1(4)$

d
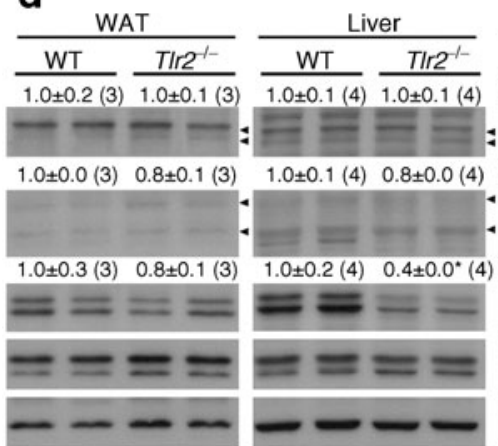

$\frac{\text { Muscle }}{\frac{\text { WT }}{\text { Tir2 } 2^{\prime-}}}$

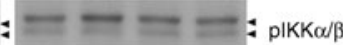

$1.0 \pm 0.1(5) \quad 0.9 \pm 0.0(4)$

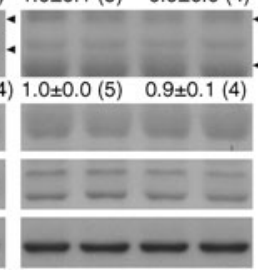

Fig. 6 Local inflammatory cytokine expression and signalling in $\mathrm{Tlr}^{-/-}$ mice. $I l l b, I l 6, T n f$ and Emr1 mRNA levels in Tlr2 ${ }^{--}(n=5-7)$ tissues relative to WT ( $n=7-8)$ of mice fed (a) RC and (b) HF diets. White bars, WT; black bars, Tlr $2^{-/}$. Immunoblot analyses on phosphorylation of IKK $\alpha / \beta$, JNK and ERK1/2 and protein content of ERK1/2 in gonadal WAT, liver and gastrocnemius muscle from WT and Tlr $2^{-/}$ mice fed (c) RC and (d) HF diets. Immunoblot analyses on

stimulated glucose uptake in $T l r 2^{-/-}$muscle. However, the differences in body weight and fat mass were not noticeable unless mice were aged up to 11 months or stimulated with a high-energy diet. The reductions in WAT mass and adipocyte size of $T l r 2^{-/-}$mice could result from the defect of pre-adipocytes differentiating into adipocytes and/or maturation of adipocytes. To address the bona fide role of TLR2 in adipocyte differentiation and maturation, we subjected embryonic fibroblasts to adipogenesis in vitro. TLR2 deficiency did not influence the abilities of

ERK $1 / 2$

Control

\section{b}

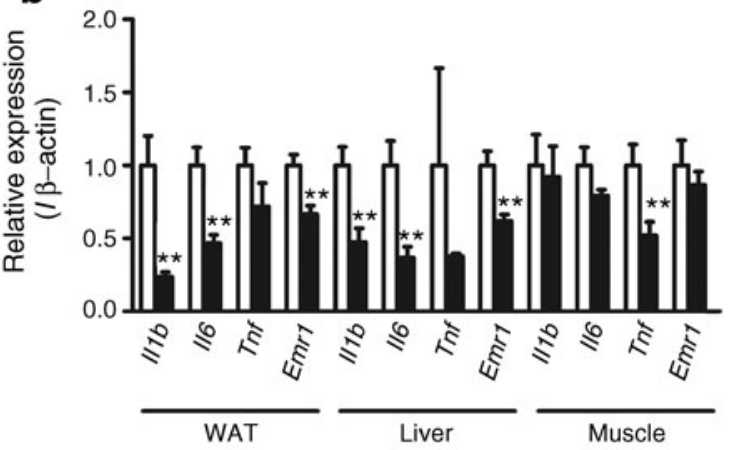

e

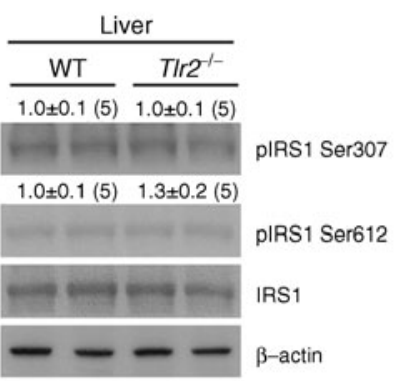

f

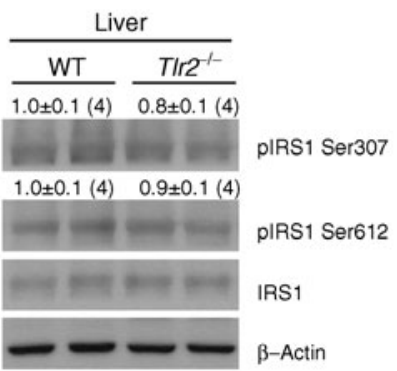

phosphorylation at Ser307 and Ser612 of IRS1 in the liver of WT and $T l r 2^{-/}$mice on (e) RC and (f) HF diets. Each band represents a tissue extract from a single mouse. The intensities of the bands, quantified densitometrically relative to WT, are shown with the sample number in parentheses. ${ }^{*} p<0.05,{ }^{* *} p<0.01$ and ${ }^{* * *} p<0.001$ for $T l r 2^{-/}$mice compared with WT mice

adipocyte differentiation and triacylglycerol accumulation, suggesting that these properties are not affected by TLR2 deficiency. Although the mechanism involved in increased energy expenditure in $\mathrm{Tlr}^{-/}$mice is not clear, the reduced deposition of excess energy in the body potentially contributes to better metabolic profiles and glucose metabolism.

Improvement of insulin sensitivity with TLR2 deficiency is present in mice fed RC, suggesting TLR2induced insulin resistance takes place in the basal 
Fig. 7 Insulin signalling in $T l r 2^{-/}$mice. Immunoblot analyses on phosphorylation at Ser473 of Akt, and detection of IRS1 phosphotyrosine level and IRS1:PI3K association by IRS1 immunoprecipitation in mice fed (a) $\mathrm{RC}$ and (b) HF diets. Each band represents a tissue extract from a single mouse. The intensities of the bands relative to WT after insulin stimulation are shown with the sample number in parentheses. Ins, insulin. ${ }^{*} p<$ 0.05 and $* * p<0.01$ for $T l r 2^{->}$ mice compared with WT mice a

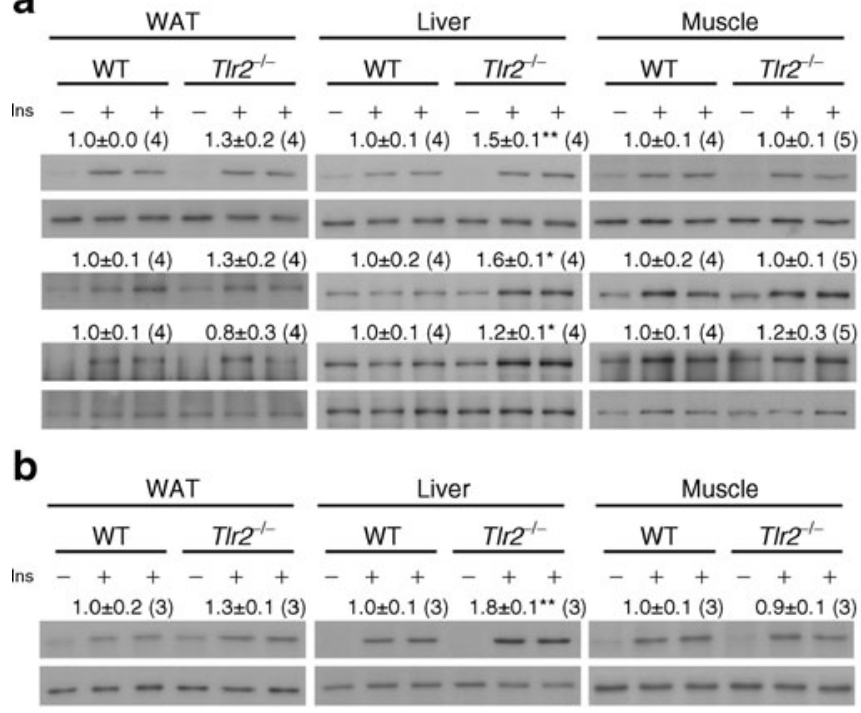

pAkt Ser473

Akt

$\begin{array}{ll}\text { IP: IRS1 IB: } p Y \\ \text { IP: IRS1 } & \text { IB: PI3K } \\ \text { IP: IRS1 } & \text { IB: IRS1 }\end{array}$

pAkt Ser473

Akt physiological condition without the need of further HF stimulation. This raises the speculation that TLR2 ligand and the signalling it mediates are present in the basal state. Although the actual endogenous TLR2 ligands have not been identified conclusively, it has been demonstrated that TLR2 recognises a large number of lipid-containing molecules [18], as well as endogenous proteins released from damaged tissues or injured cells
[29]. It is interesting to note that the critical portion of many TLR2 ligands contains a similar fatty acid component. Removal or change of these fatty acids results in loss of TLR2 activation capability [30], implicating an important role of these fatty acids in ligand recognition and receptor activation. Particularly, palmitic and stearic acids are known to be major fatty acids acylated in the lipoprotein or lipopeptide to activate TLR2 [31, 32].

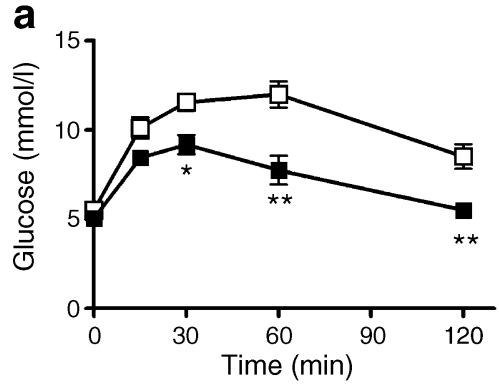

d

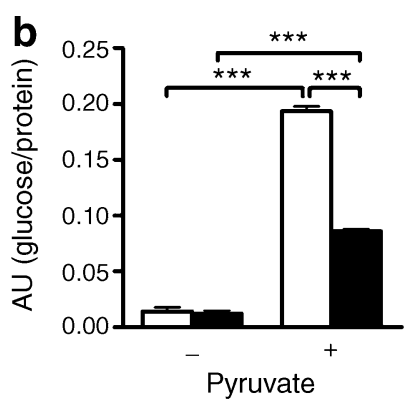

e
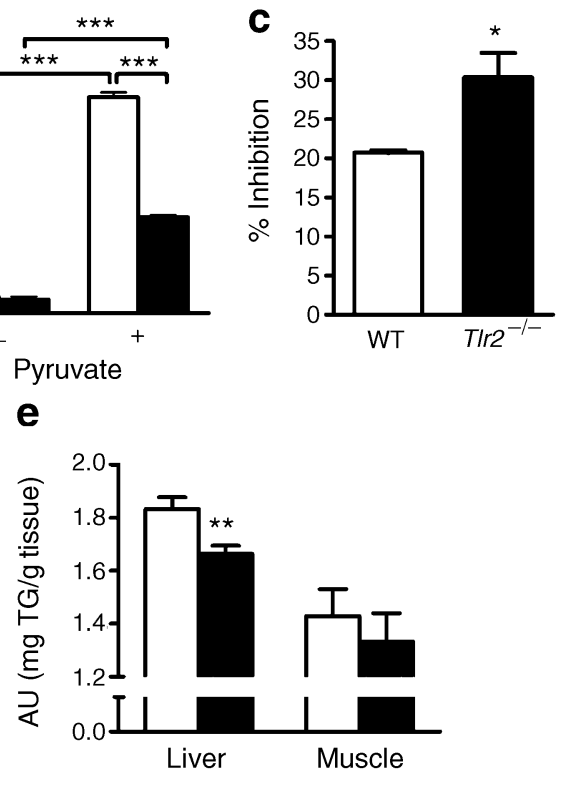

Fig. 8 Glucose metabolism and triacylglycerol content in liver and muscle of $T l r 2^{-1-}$ mice. a Plasma glucose levels during PTT in male mice fed RC ( $n=4$ each). b Basal and pyruvate-induced glucose release into the medium after $4 \mathrm{~h}$ incubation in primary hepatocytes isolated from $T l r 2^{-/}$and WT mice. c Insulin-suppressed glucose release into the medium after $16 \mathrm{~h}$ of incubation in primary hepatocytes isolated from $\mathrm{Tlr}^{-/-}$and WT mice. Results are expressed as the percentage inhibition of glucose secretion. $\mathbf{d}$ Basal and insulinstimulated glucose uptake in isolated gastrocnemius muscle from $T l r 2^{-/-}$and WT mice. Results are derived from two independent experiments performed in triplicate. e Triacylglycerol (TG) content in liver and gastrocnemius muscle of WT $(n=8)$ and Tlr $2^{-/} \quad(n=10)$ mice. White bars/symbols, WT; black bars/symbols, Tlr $2^{-/-}{ }^{*} p<0.05$, $* * p<0.01$ and $* * * p<0.001$ 
NEFA possess the capacity to induce stress/inflammatory signals not only in immune cells but also other cell types and tissues [13, 14, 33]. This phenomenon is of particular importance in conditions of nutrient excess such as obesity and diabetes or after ingestion of a fatty meal.

Inflammation-induced activation of protein kinases, such as IKK, JNK and ERK, has been shown to inhibit tyrosine phosphorylation of IRS1 [7-9] and suppress insulin action and its downstream signalling. Serine phosphorylation of IRS1 is a general mechanism to interrupt IRS1 function and insulin-signal transduction [34, 35]. For example, phosphorylation of IRS1 at Ser307 reduces IRS1 coupling to activated insulin receptors [8] and enhances IRS1 degradation [36]. Phosphorylation of IRS1 at Ser612 decreased PI3K docking to IRS1 [37]. IRS1 Ser307 can be phosphorylated by IKK and JNK [7, 8], whereas IRS1 Ser612 can be phosphorylated by ERK [9]. Our results showing enhanced insulin-stimulated Akt Ser473 phosphorylation and IRS1 tyrosine phosphorylation specifically in the liver of $T l r 2^{-/-}$ mice provide a biochemical correlate for increased hepatic insulin sensitivity. In the search for protein kinases involved in insulin signalling that are induced by inflammatory mediators, we did not detect alterations in the activation of JNK and IKK in the livers of $T l r 2^{-/}$mice. ERK activation was dramatically reduced in the livers of $T l r 2^{-/}$mice, providing a possible link between TLR2 and hepatic inflammation. However, further investigation of the hypothesised phosphorylation target on IRS1 did not reveal any change in phosphorylation of Ser307 and Ser612. These results suggest that the hypothesised phosphorylation target on IRS1 mediated through TLR2 is not Ser307 or Ser612. Nevertheless, our results implicate ERK as a downstream intracellular mediator of the TLR2-induced inflammatory response influencing the insulin signal pathway through IRS1 modification.

Our observation of improved insulin sensitivity in the absence of TLR2 supports and elaborates previous findings. For example, palmitate induced IL-6 production and inflammatory signalling, leading to inhibition of insulin-activated signal transduction through TLR2 in myotubes [21]. In addition, inhibition of TLR2 expression by TLR2 antisense oligonucleotide improves insulin sensitivity and signalling in muscle and WAT from mice fed the HF diet [38]. However, tissue specificity and transient treatment of antisense oligonucleotide limit the interpretation of long-term effect of TLR2 on complex physiological homeostasis. Although mice lacking TLR2 have recently been found to be protected from dietinduced adiposity and systemic insulin resistance [39], mechanistic insight and tissue specificity for the improvement of insulin sensitivity with TLR2 deficiency have been lacking. Collectively, these findings implicate TLR2 as a key mediator of inflammation, causing insulin resistance under conditions of nutrient excess in many key insulin-responsive tissues.

Despite the apparent upregulation of inflammatory cytokines in WAT of $o b / o b$ mice, the induction of inflammatory cytokines and TLR2 was also present in the livers of $o b / o b$ mice in our study. These findings suggest that the nutrient-induced inflammatory response and insulin resistance takes place in both WAT and liver. In support of this hypothesis, TLR2-deficiency-associated decreases in inflammatory cytokine expression and signalling and enhancement of insulin signalling were conspicuous in the liver. In contrast, the induction of inflammatory cytokines was not evident in the muscle of $o b / o b$ mice. Consistent with this, the gene expression and signalling molecules related to inflammation and insulin resistance in muscle appeared unchanged in $\mathrm{Tlr}^{-/-}$mice. Thus, the modification of peripheral insulin sensitivity through TLR2-induced inflammatory cytokine expression and signalling is likely to be mediated primarily in the liver.

Recently, the role of TLR4 in the cross-talk between nutrient-induced inflammation and insulin resistance has been investigated [40-43], and the hypothesised location for TLR4 action mediating the impairment of insulin sensitivity is predominantly in WAT. Different TLR production profiles affect the responsiveness of particular cell types and tissues to the ligand stimulation. In addition, different TLR ligands may induce activation of different downstream signalling pathways, leading to a diverse array of target gene expression and cellular responses through distinct TLRs. Given the differences in ligand preference, signalling pathways and tissue distribution between TLR2 and TLR4, it is reasonable to speculate that TLR2 and TLR4 may receive different ligand stimulation from nutrient factors and transduce inflammatory signals. Furthermore, there was no compensatory alteration in the gene expression of Tlr4 in WAT, liver and muscle (data not shown). Our results unequivocally provide TLR2 as another TLR family receptor involved in the interface of inflammatory response and metabolic disturbance.

In conclusion, our results demonstrated that TLR2 contributes to upregulation of inflammatory cytokine expression and signalling and interference of insulin action specifically in the liver. Although the detailed mechanism by which factors activate TLR2 or interact with its associated factors is not known, the results presented here represent a new paradigm that endogenous molecule(s) can utilise the innate immune receptor TLR2 to trigger the local inflammation-related signalling in the liver and subsequently affect systemic metabolism. Our findings provide a rationale for the development of the hepatic inflammatory response and insulin resistance in response to nutrient factors. 
Acknowledgements We thank A. Pendse, N. Takahashi and C.-H. Lee for discussions; Z.-H. Lin, H.-F. Jheng, the National Laboratory Animal Centre Pathology Core (C.-T. Liang), H.-T. Wu, Y.-H. Lee and the Taiwan Mouse Clinic for technical assistance; and S. Akira for kindly providing $T l r 2^{-/}$mice. This work was supported by the grant from the National Health Research Institute (NHRI-EX98-9823SC).

Duality of interest The authors declare that there is no duality of interest associated with this manuscript.

\section{References}

1. Wellen KE, Hotamisligil GS (2005) Inflammation, stress, and diabetes. J Clin Invest 115:1111-1119

2. Shoelson SE, Lee J, Goldfine AB (2006) Inflammation and insulin resistance. J Clin Invest 116:1793-1801

3. Kim JK, Kim YJ, Fillmore JJ et al (2001) Prevention of fat-induced insulin resistance by salicylate. J Clin Invest 108:437-446

4. Cai D, Yuan M, Frantz DF et al (2005) Local and systemic insulin resistance resulting from hepatic activation of IKK-beta and NFkappaB. Nat Med 11:183-190

5. Shoelson SE, Lee J, Yuan M (2003) Inflammation and the IKK beta/I kappa B/NF-kappa B axis in obesity- and diet-induced insulin resistance. Int J Obes Relat Metab Disord 27(Suppl 3): S49-S52

6. Yuan M, Konstantopoulos N, Lee J et al (2001) Reversal of obesity- and diet-induced insulin resistance with salicylates or targeted disruption of Ikk $\beta$. Science 293:1673-1677

7. Gao Z, Hwang D, Bataille F et al (2002) Serine phosphorylation of insulin receptor substrate 1 by inhibitor kappa B kinase complex. J Biol Chem 277:48115-48121

8. Aguirre V, Uchida T, Yenush L, Davis R, White MF (2000) The cJun $\mathrm{NH}(2)$-terminal kinase promotes insulin resistance during association with insulin receptor substrate-1 and phosphorylation of Ser(307). J Biol Chem 275:9047-9054

9. De Fea K, Roth RA (1997) Modulation of insulin receptor substrate-1 tyrosine phosphorylation and function by mitogenactivated protein kinase. J Biol Chem 272:31400-31406

10. Stamler R, Stamler J, Riedlinger WF, Algera G, Roberts RH (1978) Weight and blood pressure. Findings in hypertension screening of 1 million Americans. JAMA 240:1607-1610

11. Bonora E, Kiechl S, Willeit J et al (1998) Prevalence of insulin resistance in metabolic disorders: the Bruneck Study. Diabetes 47:1643-1649

12. Kahn BB, Flier JS (2000) Obesity and insulin resistance. J Clin Invest 106:473-481

13. Ghanim H, Aljada A, Hofmeyer D, Syed T, Mohanty P, Dandona P (2004) Circulating mononuclear cells in the obese are in a proinflammatory state. Circulation 110:1564-1571

14. Jove M, Planavila A, Laguna JC, Vazquez-Carrera M (2005) Palmitate-induced interleukin 6 production is mediated by protein kinase $\mathrm{C}$ and nuclear-factor kappaB activation and leads to glucose transporter 4 down-regulation in skeletal muscle cells. Endocrinology 146:3087-3095

15. Tschop M, Thomas G (2006) Fat fuels insulin resistance through Toll-like receptors. Nat Med 12:1359-1361

16. Akira S, Takeda K, Kaisho T (2001) Toll-like receptors: critical proteins linking innate and acquired immunity. Nat Immunol 2:675-680

17. Medzhitov R (2001) Toll-like receptors and innate immunity. Nat Rev Immunol 1:135-145

18. Akira S (2003) Mammalian toll-like receptors. Curr Opin Immunol 15:5-11
19. Buwitt-Beckmann U, Heine H, Wiesmuller KH, Jung G, Brock R, Ulmer AJ (2005) Lipopeptide structure determines TLR2 dependent cell activation level. FEBS J 272:6354-6364

20. Buwitt-Beckmann U, Heine H, Wiesmuller KH et al (2006) TLR1- and TLR6-independent recognition of bacterial lipopeptides. J Biol Chem 281:9049-9057

21. Senn JJ (2006) Toll-like receptor-2 is essential for the development of palmitate-induced insulin resistance in myotubes. J Biol Chem 281:26865-26875

22. Murakami K, Bujo H, Unoki H, Saito Y (2007) High fat intake induces a population of adipocytes to co-express TLR2 and TNFalpha in mice with insulin resistance. Biochem Biophys Res Commun 354:727-734

23. Nguyen MT, Favelyukis S, Nguyen AK et al (2007) A subpopulation of macrophages infiltrates hypertrophic adipose tissue and is activated by free fatty acids via Toll-like receptors 2 and 4 and JNK-dependent pathways. J Biol Chem 282:35279-35292

24. Lin Y, Lee H, Berg AH, Lisanti MP, Shapiro L, Scherer PE (2000) The lipopolysaccharide-activated toll-like receptor (TLR)-4 induces synthesis of the closely related receptor TLR-2 in adipocytes. J Biol Chem 275:24255-24263

25. Lang CH, Silvis C, Deshpande N, Nystrom G, Frost RA (2003) Endotoxin stimulates in vivo expression of inflammatory cytokines tumor necrosis factor alpha, interleukin-1beta, -6 , and highmobility-group protein-1 in skeletal muscle. Shock 19:538-546

26. Takeuchi O, Hoshino K, Kawai T et al (1999) Differential roles of TLR2 and TLR4 in recognition of gram-negative and grampositive bacterial cell wall components. Immunity 11:443-451

27. Hirosumi J, Tuncman G, Chang L et al (2002) A central role for JNK in obesity and insulin resistance. Nature 420:333-336

28. Rosen ED, Spiegelman BM (2006) Adipocytes as regulators of energy balance and glucose homeostasis. Nature 444:847-853

29. Rubartelli A, Lotze MT (2007) Inside, outside, upside down: damage-associated molecular-pattern molecules (DAMPs) and redox. Trends Immunol 28:429-436

30. Brightbill HD, Libraty DH, Krutzik SR et al (1999) Host defense mechanisms triggered by microbial lipoproteins through toll-like receptors. Science 285:732-736

31. Fujita M, Into T, Yasuda M et al (2003) Involvement of leucine residues at positions 107,112 , and 115 in a leucine-rich repeat motif of human Toll-like receptor 2 in the recognition of diacylated lipoproteins and lipopeptides and Staphylococcus aureus peptidoglycans. J Immunol 171:3675-3683

32. Muhlradt PF, Kiess M, Meyer H, Sussmuth R, Jung G (1997) Isolation, structure elucidation, and synthesis of a macrophage stimulatory lipopeptide from Mycoplasma fermentans acting at picomolar concentration. J Exp Med 185:1951-1958

33. Nguyen MT, Satoh H, Favelyukis S et al (2005) JNK and tumor necrosis factor-alpha mediate free fatty acid-induced insulin resistance in 3 T3-L1 adipocytes. J Biol Chem 280:35361-35371

34. Gual P, Le Marchand-Brustel Y, Tanti JF (2005) Positive and negative regulation of insulin signaling through IRS-1 phosphorylation. Biochimie 87:99-109

35. Taniguchi CM, Emanuelli B, Kahn CR (2006) Critical nodes in signalling pathways: insights into insulin action. Nat Rev Mol Cell Biol 7:85-96

36. Greene MW, Sakaue H, Wang L, Alessi DR, Roth RA (2003) Modulation of insulin-stimulated degradation of human insulin receptor substrate-1 by Serine 312 phosphorylation. J Biol Chem 278:8199-8211

37. Mothe I, Van Obberghen E (1996) Phosphorylation of insulin receptor substrate-1 on multiple serine residues, $612,632,662$, and 731, modulates insulin action. J Biol Chem 271:1122211227

38. Caricilli AM, Nascimento PH, Pauli JR et al (2008) Inhibition of toll-like receptor 2 expression improves insulin sensitivity and 
signaling in muscle and white adipose tissue of mice fed a high-fat diet. J Endocrinol 199:399-406

39. Himes RW, Smith CW (2010) Tlr2 is critical for diet-induced metabolic syndrome in a murine model. FASEB J 24:731-739

40. Shi H, Kokoeva MV, Inouye K, Tzameli I, Yin H, Flier JS (2006) TLR4 links innate immunity and fatty acid-induced insulin resistance. J Clin Invest 116:3015-3025

41. Tsukumo DM, Carvalho-Filho MA, Carvalheira JB et al (2007) Loss-of-function mutation in Toll-like receptor 4 prevents diet-induced obesity and insulin resistance. Diabetes 56:1986-1998

42. Poggi M, Bastelica D, Gual P et al (2007) C3H/HeJ mice carrying a toll-like receptor 4 mutation are protected against the development of insulin resistance in white adipose tissue in response to a high-fat diet. Diabetologia 50:1267-1276

43. Kim F, Pham M, Luttrell I et al (2007) Toll-like receptor-4 mediates vascular inflammation and insulin resistance in dietinduced obesity. Circ Res 100:1589-1596 